\title{
Editorial
}

\author{
Ercan E. Kuruoglu \\ Istituto di Scienza e Tecnologie dell'Informazione “A. Faedo" (ISTI), Area della ricerca CNR di Pisa, \\ via G. Moruzzi 1, 56124 Pisa, Italy \\ Email: kuruoglu@isti.cnr.it
}

\section{Carlo Baccigalupi}

Scuola Internazionale Superiore di Studi Avanzati (SISSA/ISAS), via Beirut 4, 34014 Trieste, Italy

Email: bacci@sissa.it

We live in an epoch where the frontiers of our investigation and comprehension of fundamental physics depend largely on the light coming from the sky, that is, on the study of galactic and extra-galactic radiation. Watching the sky, in principle, we have access to the highest energies conceivable, generated by the laws of nature in extreme conditions, such as nearby black holes or even close to the origin of the universe itself. For example, in the microwave band, the extra-Galactic radiation is dominated by a markedly isotropic component, obeying a black body spectrum characterized by a temperature of about 2.726 Kelvin. That is the relic of the Big Bang, originated just 300000 years after the initial starting point of the universe. This radiation, namely the cosmic microwave background (CMB) radiation, today is the most important observable we have to access the mysterious physics of the Big Bang itself. The latter is telling us about the unknown fundamental interactions and particles, the physics of spacetime, and the nature of quantum gravity, and represents the only way to address those issues in physics today. Electronics hardware technology has reached in these very recent years the capability to study the tiniest details of the $\mathrm{CMB}$, carrying the image of the primordial stage of cosmic geometry, structure, and composition. Such a fantastic challenge is ongoing in this very moment, while several CMB detectors are operating and advanced probes are being designed for the forthcoming decades.

Many breakthroughs in physics are made possible by the use of the most advanced data analysis techniques. The present datasets obtained in astrophysical and cosmological observations are huge, and cover the entire electromagnetic spectrum, dealing with very different processes, from gamma and X-rays of the high-energy astrophysics of compact stars or black holes, to the microwave and infrared emission from the whole large-scale universe. This variety of the observational techniques and signals to deal with represents a formidable challenge for signal processing. We need state-ofthe-art techniques that can analyse, summarise, and extract the necessary information from this ocean of data.

To continue with the example above, the microwave sky is dominated by the $\mathrm{CMB}$ radiation, but several processes contribute to the total emission, coming for instance from all the processes occurring along the line of sight, such as the emission from other galaxies or clusters of those, as well as from the diffuse gas in our own Galaxy. Each of these processes are most relevant in different contexts in astrophysics and cosmology. Recently, the astrophysics field has benefited a great deal from the rich research work going on source separation in the signal processing field. Source separation aims at the recovery of the various different components from the multiband observations exploiting the differences between them, induced by their independent physical origins.

Despite the mutual interest, the two disciplines suffer from lack of a common publication ground, implying that the results produced in one of them are not immediately visible in the other. The aim of the present issue is to provide a unified platform that would strengthen the bridge between signal processing and astrophysics and cosmology and enable the sharing of information. We would like to provide astrophysicists and cosmologists with a spectrum of the most advanced signal processing techniques and the signal processing community an exposure to various vital real problems in analysing astrophysics data that await solution. Finally, our aim is to provide a reference for present and future literature, in the widest possible context, accounting for various applications and algorithms proposed. Indeed, as the reader may see, the topics we collected range from solar physics, thus on the scale of stars, to the reconstructuon of the most ambitious signal from the Big Bang, with the reconstruction of the CMB pattern on all sky. The methods presented in the issue range from transform domain analysis of such wavelets to data mining techniques. 
We start the special issue with four papers addressing the problem of separating components in astrophysical radiation maps which is a very hot problem due to the recent availability of WMAP satellite radiation maps and the future Planck satellite mission. The first paper on the problem is by Bedini et al. who consider the separation of components which are mutually dependent, therefore differing from classical ICA approaches. Therefore the paper also houses novelties from a signal processing point of view. The second paper in the sequel, by Forni and Aghanim, considers a more specific problem, namely the separation of kinetic SZ signal from primary CMB fluctuations. The next paper, by López-Caniego et al., provides instead a technique based on Bayesian detection theory for the separation of point sources from the rest of the astrophysical radiation map. Moudden et al., in contrast to these works, consider separation in the transform domain. In particular, they propose a new separation method in the wavelet space.

A related paper, by Starck et al., again in the wavelet methodological frame follows: it analyses the spatial distribution of the galaxies by multiscale methods. Jin et al. also concentrate their efforts on the analysis of the statistical distribution of the CMB utilising wavelet transform, with the particular aim of detecting non-Gaussianity. Continuing with transform domain analysis techniques, closely related to wavelet ideas, Thiebaut and Roques present time-frequency analysis techniques applied to irregularly sampled astronomical time series.

Astrophysical images are obtained by mechanisms far from perfect and the images are corrupted with noise and are blurred by the cameras' limited resolution and may be distorted by nonlinearities in the cameras. Lantéri and Theys provide a technique for the restoration of the images in the case of Poisson data and additive Gaussian noise.

Recent satellite missions have provided us with vast amounts of data. Therefore, it is of paramount importance to build efficient indexing methods and equally design data mining methods to recover information from big databases. To this end, Frailis et al. propose a multidimensional indexing method. Again due to the vast amount of data available, it is important to be able to store them in an economic way. Lastri et al. suggest a new compression technique which is virtually lossless.

Bratsolis tackles the problem of classification of astrophysical images and his paper gives a flavour of the application of neural-network-based techniques to the problem. Flitti et al. deal with the problem of 3D segmentation and present a new technique which they apply on real data. Wilson et al. present the adaptive DFT-based interferometer tracking algorithm they have designed.

While most of the works presented here are on cosmological problems of dimensions, surely solar science, in which important developments take place, is also in the interest area of the issue. In particular, Zharkov et al. present a detailed system for the automated recognition of sunspots. They use a combination of elaborate morphological operators which make the paper interesting also from an image processing point of view.
Lastly, to add a flavour of implementation issues of digital signal processing systems for astrophysical tasks we included a work by Belbachir et al. who describe a DSP system for infrared astronomy that implements a combination of lossy and lossless compression.

We would like to thank all of the authors who contributed to this issue for their very interesting work. We are very grateful to the referees who gave their time and energy in the review process and contributed immensely to the value of this issue. Finally, we would like to thank the Editor-in-Chief Dr. Moonen and the EURASIP JASP staff for helping us at every stage of the creation of this issue. We do hope that the reader will find the issue useful, interesting, and inspirational.

\section{Ercan E. Kuruoglu Carlo Baccigalupi}

Ercan E. Kuruoglu was born in Ankara, Turkey, in 1969. He obtained his B.S. and M.S. degrees both in electrical and electronics engineering from Bilkent University in 1991 and 1993, respectively. He completed his graduate studies with M.Phil. and Ph.D. degrees in information engineering at the Cambridge University, in the Signal Processing Laboratory, in 1995 and 1998, respectively. Upon graduation from Cam-

bridge, he joined the Xerox Research Center in Cambridge as a permanent member of the Collaborative Multimedia Systems Group. After two years in Xerox, he won an ERCIM fellowship which he spent in INRIA-Sophia Antipolis, France, and IEI CNR, Pisa, Italy. In January 2002, he joined ISTI-CNR, Pisa, as a permanent member. His research interests are in statistical signal processing and information and coding theory with applications in image processing, astronomy, telecommunications, intelligent user interfaces, and bioinformatics. He is currently on the Editorial Board of Digital Signal Processing and an Associate Editor for the IEEE Transactions on Signal Processing. He was the Guest Editor for a special issue of the Signal Processing Journal on "Signal Processing with Heavy-tailed Distributions," December 2002. He is the Special Sessions Chair for EURASIP European Signal Processing Conference, EUSIPCO 2005, and is the Technical Chair for EUSIPCO 2006. He is also a Member of the IEEE Technical Committee on Signal Processing Theory and Methods. He has more than 50 publications and holds 5 US, European, and Japanese patents.

Carlo Baccigalupi is currently an Assistant Professor at SISSA/ISAS. He is a member of the Planck and EBEx cosmic microwave background (CMB) polarization experiment. In Planck, he is leading the working group on component separation, and in EBEx he is responsible for the control of the foreground polarized contamination to the $\mathrm{CMB}$ radiation. He is the author of about 40 papers on refereed international scientific review, on topics ranging from the theory of gravity to $\mathrm{CMB}$ data analysis. He is teaching linear cosmological perturbations and CMB anisotropies courses for the Astroparticle Ph.D. course at SISSA. 
He is involved in long-term international projects. The most important ones are the Long-term Space Astrophysics funded by NASA for the duration of five years, on component separation on COBE, WMAP, and future CMB experiments, and a one-year mercator professorship to be carried out in the University of Heidelberg in the academic year 2005/2006. 